\title{
An education and training programme for radiological institutes: impact on the reduction of the $\mathrm{CT}$ radiation dose
}

\author{
Sebastian T. Schindera $\cdot$ Reto Treier • \\ Gabriel von Allmen • Claude Nauer • Philipp R. Trueb • \\ Peter Vock $\cdot$ Zsolt Szucs-Farkas
}

Received: 22 February 2011 /Revised: 14 April 2011 / Accepted: 15 April 2011 /Published online: 31 May 2011

(C) European Society of Radiology 2011

\begin{abstract}
Objectives To establish an education and training programme for the reduction of $\mathrm{CT}$ radiation doses and to assess this programme's efficacy.

Methods Ten radiological institutes were counselled. The optimisation programme included a small group workshop and a lecture on radiation dose reduction strategies. The radiation dose used for five $\mathrm{CT}$ protocols (paranasal sinuses, brain, chest, pulmonary angiography and abdomen) was assessed using the dose-length product (DLP) before and after the optimisation programme. The mean DLP values were compared with national diagnostic reference levels (DRLs).

Results The average reduction of the DLP after optimisation was $37 \%$ for the sinuses ( 180 vs. $113 \mathrm{mGycm}, P<$ $0.001), 9 \%$ for the brain (982 vs. $896 \mathrm{mGycm}, P<0.05$ ), $24 \%$ for the chest ( 425 vs. $322 \mathrm{mGycm}, P<0.05$ ) and $42 \%$ for the pulmonary arteries ( 352 vs. $203 \mathrm{mGycm}, P<0.001$ ). No significant change in DLP was found for abdominal CT. The post-optimisation DLP values of the sinuses, brain,
\end{abstract}

S. T. Schindera $(\bowtie) \cdot$ G. von Allmen $\cdot$ P. Vock $\cdot$ Z. Szucs-Farkas University Institute of Diagnostic, Interventional and Pediatric Radiology, University Hospital Berne, University of Berne, Freiburgstrasse,

CH-3010 Berne, Switzerland

e-mail: sschindera@aol.com

R. Treier $\cdot$ P. R. Trueb

Radiation Protection Division, Federal Office of Public Health, Berne, Switzerland

\section{Nauer}

University Institute of Diagnostic and Interventional

Neuroradiology, University Hospital Berne, University of Berne, Berne, Switzerland chest, pulmonary arteries and abdomen were $68 \%, 10 \%$, $20 \%, 55 \%$ and $15 \%$ below the DRL, respectively.

Conclusions The education and training programme for radiological institutes is effective in achieving a substantial reduction in $\mathrm{CT}$ radiation dose.

Keywords Computed tomography Radiation dose . Training programme $\cdot$ Protocol optimisation $\cdot$ Diagnostic reference level

\section{Introduction}

Since its introduction in the early 1970s, computed tomography (CT) has evolved into one of the most important imaging techniques in medicine. The frequency of CT examinations has tremendously increased during the most recent decade mainly due to technical advances in multidetector CT and its wide availability. As a consequence of the increasing use of $\mathrm{CT}$, exposure of the population to ionising radiation has also grown substantially. Because ionising radiation has carcinogenic effects, the number of radiation-induced cancers will likely increase over time as well. Brenner and Hall have estimated that $1.5 \%$ to $2.0 \%$ of all future cancers in the United States will be attributable to the radiation exposure from CT [1]. In absolute numbers, this estimation translates to an annual increase of approximately 29,000 additional tumours in the United States [2]. Based on these alarming estimations, the radiology community (radiologists, technicians, medical physicists and CT manufacturers) must make every possible effort to minimise the $\mathrm{CT}$ radiation dose for patients.

In the most recent decade, CT manufacturers have developed several techniques for reducing radiation doses, 
such as automatic tube current modulation or iterative reconstruction algorithms [3-6]. At the same time, radiologists and medical physicists have published many scientific manuscripts regarding low-dose CT protocols and general dose reduction strategies [7-15]. However, the various techniques and strategies for dose reduction are only effective if they are applied correctly. Unfortunately, the promotion of education and training programmes on dose optimisation for radiologists and technologists has been neglected in the past. To date, many radiologists and technologists have been unaware of basic optimisation techniques to reduce the $\mathrm{CT}$ radiation dose [16]. On the basis of this insufficient knowledge, we hypothesised that an education and training programme for radiological institutes would result in a significant reduction of the CT radiation dose to the patient.

The objective of our study was therefore to establish an education and training programme for radiologists and technologists regarding the reduction of $\mathrm{CT}$ radiation doses and to assess this programme's efficacy.

\section{Materials and methods}

This study complied with the Health Insurance Portability and Accountability Act and was approved by our institutional review board. In addition, the requirement for informed consent was waived. The study was fully funded by the Federal Office of Public Health (FOPH) of Switzerland.

In January 2009, a CT radiation consulting team that consisted of three radiologists (S.T.S., C.N., Z.S.) and a radiological technologist (G. A.) was established. All members of the CT radiation consulting team were working full time in a university hospital that had more than 1,000 beds. The radiologists had broad experience in the optimisation of $\mathrm{CT}$ radiation doses, gained from a multitude of clinical and scientific projects. The three radiologists were working as attending physicians in the fields of neuroradiolgical, thoracic and abdominal imaging. The radiological technologist was the chief technologist for $\mathrm{CT}$ at the university hospital and had been involved in various clinical and scientific dose optimisation projects in the past. In addition, two medical physicists in the radiation protection division of the FOPH played advisory roles in this project.

In November 2009, 29 radiological institutes were invited by letter to participate in an education and training programme for CT dose optimisation. Only radiological institutes that were accessible within $2 \mathrm{~h}$ by car and were located in Switzerland were selected. Participation in the education and training programme was voluntary and free of charge. The invitees were informed that the collected data would remain strictly confidential and anonymous. Optimisation of the CT protocols was restricted to $\mathrm{CT}$ systems from Siemens Healthcare (Forchheim, Germany) or GE Healthcare (Milwaukee, WI, USA) because the members of the radiation consulting team had experience with these types of $\mathrm{CT}$ and our objective was to provide the most up-to-date, manufacturer-specific dose reduction strategies. Furthermore, these manufacturers were willing to support the radiation consulting team with technical advice.

Of the 29 invited institutes, 12 were interested in our education and training programme. Of these 12 institutes, ten were chosen randomly to participate in this study. These participating institutes included three private radiological institutes and seven radiological departments in public hospitals (number of beds: 100-535). Six of these institutes operated a CT system from Siemens Healthcare, and four institutes operated one from GE Healthcare (Table 1).

In January and February 2010, one radiologist (S.T.S.) of the radiation consulting team visited all of the participating institutes to explain the processes involved in the project and to collect data regarding the relevant technical parameters of the most frequently used adult CT protocols (e.g. the tube voltage, tube current-time product, pitch, collimation and use of automatic tube current modulation).

The consulting service consisted of three phases: the control period, the education and training period, and the follow-up period. During the control and follow-up periods, the radiation doses of the patients who had undergone clinically indicated CT examinations of the brain, paranasal sinuses, chest, pulmonary arteries or the abdomen were recorded. The CT examinations of the brain were unenhanced studies to rule out the presence of intracerebral haemorrhage and cerebral ischaemia. The indications for $\mathrm{CT}$ of the paranasal sinuses included inflammatory diseases, mid-facial traumas or preoperative work-ups. Chest CT consisted of routine contrast-enhanced imaging to diagnose infectious pulmonary diseases, lymph nodes or thoracic tumours. CT of the pulmonary arteries was applied to confirm or rule out pulmonary embolisms. CT examinations of the abdomen included the pelvis and were performed during the portal-venous phase of the contrast enhancement. The indications for abdominal CT included the presence of infectious diseases, tumours, lymph nodes

Table 1 The CT types used in the ten radiological institutes studied

\begin{tabular}{ll}
\hline CT system from Siemens Healthcare & $\begin{array}{l}\text { CT system from } \\
\text { GE Healthcare }\end{array}$ \\
\hline Somatom sensation $16(n=1)$ & Lightspeed $16(n=3)$ \\
Somatom emotion $16(n=1)$ & Lightspeed $64(n=1)$ \\
Somatom sensation $40(n=2)$ & \\
Somatom sensation $64(n=1)$ & \\
Somatom definition, dual source $(n=1)$ & \\
\hline
\end{tabular}


and pre- and post-operative work-ups. The patient inclusion criteria included a minimum age of 18 years and an average body mass index (BMI) of between 18.5 and 25 . There was no minimum or maximum number of patients for whom data had to be collected by the participating institutes during the control and follow-up periods. Not all institutes provided radiation dose data for each of the five $\mathrm{CT}$ protocols. The radiation dose, which was expressed as the dose-length product (DLP), was displayed on the CT console and stored in the local database at the end of each examination. DLP measurements were not performed. There may be differences between the measured and displayed dose values but the expected agreement between the two sets of data are reasonable so that displayed dose values are probably sufficiently accurate [17]. The radiological technicians were advised regarding the method to collect and arrange the radiation dose data in a pre-defined table template. Data collection lasted 8 weeks during the control and follow-up periods.

During the education and training periods, each of the institutes was consulted by a radiologist (S.T.S.) and a radiological technician (G. A.) of the radiation consulting team. The consultations took place at each institute and included an advising session on CT protocol optimisation and a lecture on $\mathrm{CT}$ radiation protection issues and strategies for reducing the $\mathrm{CT}$ radiation dose. During the individual advising session on the $\mathrm{CT}$ protocols, the radiation consulting team made suggestions regarding the optimisation of technical parameters and application of different contrast enhancement phases. The consultation was conducted as a face-to-face, small group workshop and was attended by a radiologist and a radiological technologist who were responsible for optimising the CT protocols at their respective institutes. Recommended optimisations are widely accepted in the medical literature and can be applied independently of the configuration of the CT system (Table 2). The institutes were under no obligation

Table 2 Recommendations for the optimisation of CT protocols

- Application of reduced tube voltage for CT angiography (100 or $80 \mathrm{kVp})$ :

- cerebral CT angiography [30, 31]

- pulmonary CT angiography [32-34]

- aorto-iliac CT angiography $[8,35,36]$

- Lowering of the tube voltage and/or tube current for non-contrast CT [37]

- Reduction of the total number of imaging phases (e.g. split-bolus technique) $[38,39]$

- Adjustment of image quality reference values of the automatic tube current modulation for oversized patients [40]

- Reduction of the tube voltage and/or tube current for survey CT [41, 42]

- Positioning of the X-ray tube below the table for the survey CT (e.g. $180^{\circ}$, postero-anterior position) [41, 42]

- Prevention of the routine use of narrow collimations (e.g. $<1 \mathrm{~mm}$ ) for $\mathrm{CT}$ of the thorax or abdomen $[37,43]$ to accept the recommendations that were made. The lecture, which was targeted at all of the radiologists and radiological technologists of the institutes, provided information on radiobiology, the cancer risk that is associated with $\mathrm{CT}$ examinations, CT dosimetry and practical advice for reducing $\mathrm{CT}$ radiation doses (Table 3 ). The on-site consultation lasted for $3 \mathrm{~h} ; 2 \mathrm{~h}$ were used for the individual sessions advising on CT protocol optimisation, and $1 \mathrm{~h}$ was used for the lecture.

The mean DLP values before and after the training regarding a specific CT protocol were compared using a Wilcoxon matched-pairs test for each institute. An analysis of variance with additional post-hoc tests was used to analyse the pooled data for all of the institutes. Because the radiation doses exhibited significant differences between the institutes, especially for the doses before the consultations, we used the same number of $\mathrm{CT}$ examinations from each institute before and after the training for a given CT protocol. The statistical tests were performed using statistical software (StatSoft Inc., Tulsa, OK, USA) and MedCalc software (MedCalc, Mariakerke, Belgium). $P$ values of less than 0.05 were considered statistically significant. In addition, the DLPs from the institutes were compared with the national diagnostic reference levels (DRLs) of Switzerland [18]. The effective dose was estimated by multiplying the DLP by $0.0022 \mathrm{mSv} / \mathrm{mGycm}$ for the head imaging, by $0.017 \mathrm{mSv} / \mathrm{mGycm}$ for the thoracic imaging and by $0.016 \mathrm{mSv} / \mathrm{mGycm}$ for the abdominal imaging [19]. The used DLP conversion factors did not consider the new tissue weighting factors published by the International Commission on Radiological Protection [20].

\section{Results}

During both the control and follow-up periods, the DLP values from $180 \mathrm{CT}$ examinations were collected (24 CT examinations of the paranasal sinuses, 43 of the brain, 45 of the chest, 21 of the pulmonary arteries, and 47 of the abdomen).

After the education and training programme, a decrease in the $\mathrm{CT}$ radiation dose was achieved for the paranasal sinuses,

Table 3 Practical strategies for reducing CT radiation doses

- Review the clinical justification for the CT examination and reconsider using imaging techniques that are free of ionising radiation (e.g. MRI, ultrasound) [29]

- Limit the imaging range to the area of interest, for follow-up studies in particular [44]

- Prevent the extend beyond the anatomic limits (e.g., thorax, abdomen) which does not add clinically important information [7]

- Position the arms above the shoulders during chest CT [45]

- Position the patient precisely in the gantry [12, 46, 47] 
brain, chest and pulmonary arteries (Table 4 ). Compared with the control period, the average reduction in the DLP in the follow-up period was $37 \%$ for the sinuses $(P<0.001), 9 \%$ for the brain $(P<0.05), 24 \%$ for the chest $(P<0.05)$, and $42 \%$ for the pulmonary arteries $(P<0.001)$. No significant difference was observed for the DLP values of the abdomen $(P=0.60)$. The average post-optimisation DLP values were lower than the corresponding DRLs (sinuses: $-68 \%$, brain: $-10 \%$, chest: $-20 \%$, pulmonary arteries: $-55 \%$ and abdomen: $-15 \%$; Table 4). Except for the CT examinations of the chest, the average pre-optimisation DLP values were already slightly lower than the DRL values.

The estimated effective dose decreased from 0.4 to $0.3 \mathrm{mSv}, 2.2$ to $2.0 \mathrm{mSv}, 7.2$ to $5.5 \mathrm{mSv}$, and 6.0 to $3.5 \mathrm{mSv}$ for the $\mathrm{CT}$ of the sinuses, brain, chest and pulmonary arteries, respectively $(P<0.05)$. For the $\mathrm{CT}$ of the abdomen, the effective dose was $8.6 \mathrm{mSv}$ for the control period and $8.9 \mathrm{mSv}$ for the follow-up period.

A slight increase in DLP values was observed in the followup period (compared with the control period) with regard to $\mathrm{CT}$ of the sinuses in one of the radiological institutes and with regard to CT of the brain in another radiological institute (Fig. 1a, b). In several radiological institutes, the DLP values for the CT of the abdomen were larger during the follow-up period than the control period (Fig. 1e). A marked variation in the DLP values existed among the radiological institutes for the same CT protocol (Fig. 1a-e). For example, the radiation doses of the $\mathrm{CT}$ protocols for the pulmonary arteries differed by a factor of up to 3.5 among the institutes before the optimisation programme was applied (Fig. 1d). After the optimisation programme, the differences in the DLP values among the institutes (shown by the standard deviations as well as the 5th and 95th percentiles) reduced substantially for the five different CT protocols (Table 4).

\section{Discussion}

The results of our study demonstrate the effectiveness of a voluntary education and training programme in reducing the $\mathrm{CT}$ radiation dose in several radiological institutes. The largest decrease in the radiation dose used in cranial imaging was achieved for CT of the paranasal sinuses (up to $37 \%$ ). The largest dose decrease for the thoracic imaging was achieved for the CT of the pulmonary arteries (up to $42 \%)$. However, no reduction of the radiation dose was achieved after the education and training programme for abdominal CT. A possible explanation for this finding might be the radiologists' concerns about suboptimal image quality resulting from lower dose CT protocols, possibly leading to a greater risk of overlooking low contrast lesions [21-24]. Conversely, a marked reduction in the radiation dose is feasible in thoracic $\mathrm{CT}$ imaging because the increase in image noise is compensated for by the inherent contrast between air (lower attenuation) and the pulmonary vessels and interstitial structures (higher attenuation).

Participation in our education and training programme for CT dose optimisation was voluntary. Therefore, radiological institutes that participated in this study were motivated to actively reduce the CT radiation dose, which resulted in a very productive collaborative counselling process. The participation of these motivated institutes is reflected by the fact that the average DLP values were lower before the optimisation process in many institutes than the DRL values. We assume that a mandatory approach for education and training would have resulted in a less significant dose reduction. Institutes with little to no interest in reducing CT radiation doses should first be sensitised to the need for this process using an awareness campaign. Recently, awareness campaigns for paediatric and adult CT imaging have been launched in the United States $[25,26]$. Two recent investigations have confirmed our findings that educational initiatives can successfully reduce the CT radiation dose [27, 28]. Raff and coworkers reported an average reduction of $53 \%$ in the radiation dose for cardiac $\mathrm{CT}$ in 15 radiological institutes by adopting a best-practice CT model [27]. This best-practice CT model included a minimised imaging range, a heart rate reduction, electrocardiographic-gated tube current modulation, and reduced tube voltage in patients, where applicable. In

Table 4 Mean CT radiation doses of the ten radiological institutes during the control and follow-up periods. The national DRLs are included as a reference

\begin{tabular}{|c|c|c|c|c|c|}
\hline $\mathrm{CT}$ examination & $\mathrm{DRL}[\mathrm{mGycm}]$ & $\begin{array}{l}\text { Dose during the control } \\
\text { period }[\mathrm{mGycm}]\end{array}$ & $\begin{array}{l}\text { Dose during the follow-up } \\
\text { period }[\mathrm{mGycm}]\end{array}$ & $\begin{array}{l}\text { Percentage } \\
\text { change }\end{array}$ & $P$ Value \\
\hline Paranasal sinuses & 350 & $180 \pm 59(155,205)$ & $113 \pm 36(98,128)$ & $-37 \%$ & $<0.001$ \\
\hline Brain & 1,000 & $982 \pm 200(920,1,043)$ & $896 \pm 177(841,950)$ & $-9 \%$ & $<0.05$ \\
\hline Chest & 400 & $425 \pm 213(361,489)$ & $322 \pm 116(287,356)$ & $-24 \%$ & $<0.01$ \\
\hline Pulmonary arteries & 450 & $352 \pm 172(273,431)$ & $203 \pm 61(176,231)$ & $-42 \%$ & $<0.001$ \\
\hline Abdomen & 650 & $539 \pm 174(488,590)$ & $555 \pm 118(521,590)$ & $+3 \%$ & $=0.60$ \\
\hline
\end{tabular}

The data on the dose was assessed by the DLP. The data are presented as means, with the 5 th and 95th percentiles in parentheses 
Fig. 1 Dose-length product values acquired during the control and follow-up periods for each radiological institute and the various CT protocols [a CT of the paranasal sinuses, b CT of the brain, $\mathbf{c}$ CT of the chest, $\mathbf{d} \mathrm{CT}$ of the pulmonary arteries, and e CT of the abdomen]. Not all institutes provided radiation dose data for each of the five CT protocols. Therefore, not all ten institutes are shown in each figure. The straight horizontal line in each figure represents the corresponding national DRL values
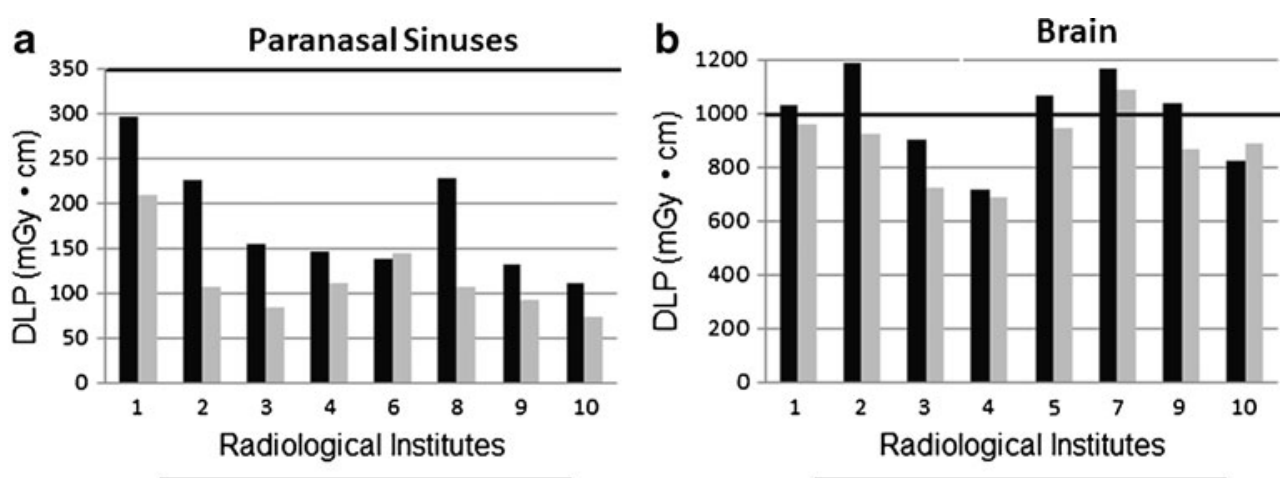

- control period $=$ follow-up period
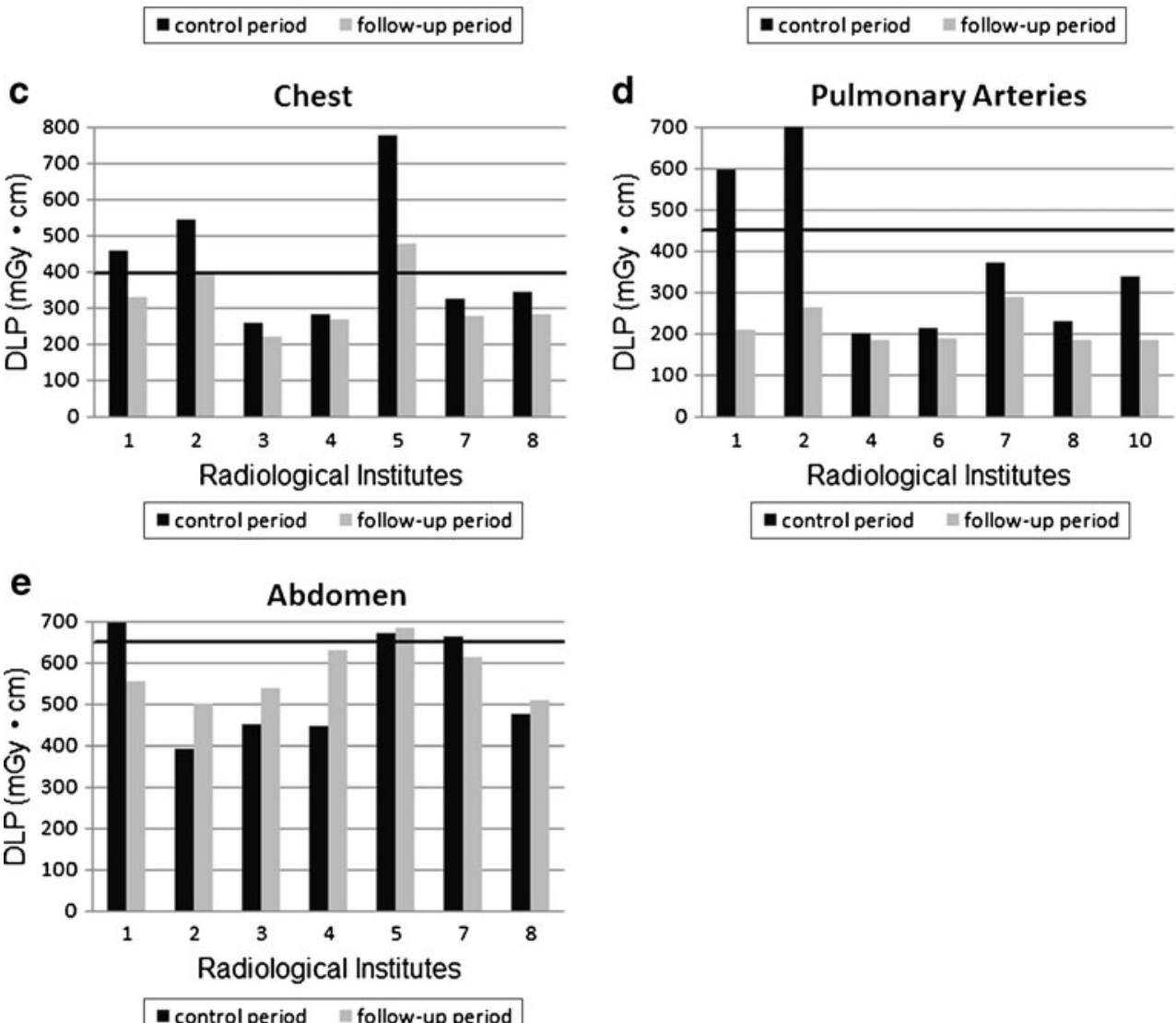

another study by Wallace et al., the radiation doses used in four common adult CT protocols (unenhanced CT of the brain, CT pulmonary angiography, unenhanced CT of the lumbar spine and CT urography) decreased substantially in radiological institutes that participated in a 1-day optimisation training workshop [28]. An average dose reduction of $46 \%$ for brain CT and 28\% for pulmonary CT angiography was achieved. One key factor in obtaining these significant dose reductions in the two previous studies and in our study was the individual supervision of the participating institutes by an on-site radiation consulting team. Based on the results of our study, we decided to expand the education and training programme to all of the interested radiological institutes in Switzerland and to institutes working with CT systems from all manufacturers. A specially designed webpage (www.ct-consulting.ch) provides relevant information from our service and allows communication with the members of our radiation consulting team and service reservation.

Reduction of the CT radiation dose requires a multifaceted approach, as suggested by Hricak et al. [29]. Three major components have to be considered for successful dose optimisation in CT: (a) implementation of dosereduction techniques, (b) use of clinical decision guidelines and (c) application of education and training programmes. Currently, very effective dose reduction techniques are available, and various clinical decision guidelines have been published by several national societies (e.g. the American College of Radiology and the Royal College of Radiologists) for the appropriate use of CT. Furthermore, 
several educational resources, such as review articles and websites describing radiation dose reduction, exist for the use of radiologists and technologists. However, on-site, face-to-face training workshops have not yet been widely organised. We believe that to achieve successful radiation dose reduction, each institute should be advised on an individual basis. The consulting service may be provided by either application specialists from a CT manufacturer or experts from a national radiological society or a governmental agency.

Several potential limitations of our study merit consideration. First, an evaluation of the diagnostic image quality of the CT protocols was not performed during the control and follow-up periods. However, the dose reduction strategies were adopted only when the image quality was deemed sufficient by the radiologists at the participating institutes. Second, the direct assessment of an individual dose-saving recommendation on the overall dose reduction for a specific CT protocol was not possible because multiple measures were implemented simultaneously. Furthermore, factors other than the recommendations made by our radiation consulting team may have affected the dose reduction in the follow-up period. Third, a standardised calibration of the CT dose index for each CT system was not performed before the dose data were collected. However, no adjustments were made on the CT units during the study period, and each institute contributed the same number of radiation dose values in the control and follow-up periods. Therefore, any systematic errors in the values of CT dose index are unlikely to have affected the results of this study. Fourth, only the radiation doses of patients who had an average BMI were assessed so that we would have a homogeneous group of patients to compare in the control and follow-up periods. Thus, it is not clear whether a major dose reduction can be expected in overweight or obese patients while maintaining diagnostic accuracy.

In conclusion, CT radiation dose can be substantially reduced using an on-site education and training programme for radiologists and technologists. The largest dose reductions can be expected in the head and thorax. Future studies are needed to assess the state- or nation-wide implementation of an education and training programme to reduce $\mathrm{CT}$ radiation dose.

Acknowledgement The authors thank the members of the participating radiological institutes for their great effort.

\section{References}

1. Brenner DJ, Hall EJ (2007) Computed tomography-an increasing source of radiation exposure. N Engl J Med 357:2277-2284
2. Berrington de Gonzalez A, Mahesh M, Kim KP et al (2009) Projected cancer risks from computed tomographic scans performed in the United States in 2007. Arch Intern Med 169:2071-2077

3. Marin D, Nelson RC, Schindera ST et al (2010) Low-tubevoltage, high-tube-current multidetector abdominal $\mathrm{CT}$ : improved image quality and decreased radiation dose with adaptive statistical iterative reconstruction algorithm-initial clinical experience. Radiology 254:145-153

4. Kalra MK, Maher MM, Toth TL et al (2004) Techniques and applications of automatic tube current modulation for CT. Radiology 233:649-657

5. McCollough CH, Bruesewitz MR, Kofler JM Jr (2006) CT dose reduction and dose management tools: overview of available options. Radiographics 26:503-512

6. Hara AK, Paden RG, Silva AC, Kujak JL, Lawder HJ, Pavlicek $\mathrm{W}$ (2009) Iterative reconstruction technique for reducing body radiation dose at CT: feasibility study. AJR Am J Roentgenol 193:764-771

7. Campbell J, Kalra MK, Rizzo S, Maher MM, Shepard JA (2005) Scanning beyond anatomic limits of the thorax in chest CT: findings, radiation dose, and automatic tube current modulation. AJR Am J Roentgenol 185:1525-1530

8. Schindera ST, Graca P, Patak MA et al (2009) Thoracoabdominalaortoiliac multidetector-row CT angiography at 80 and $100 \mathrm{kVp}$ : assessment of image quality and radiation dose. Invest Radiol 44:650-655

9. Szucs-Farkas Z, Kurmann L, Strautz T, Patak MA, Vock P, Schindera ST (2008) Patient exposure and image quality of lowdose pulmonary computed tomography angiography: comparison of $100-$ and $80-\mathrm{kVp}$ protocols. Invest Radiol 43:871-876

10. Nakayama Y, Awai K, Funama Y et al (2005) Abdominal CT with low tube voltage: preliminary observations about radiation dose, contrast enhancement, image quality, and noise. Radiology 237:945-951

11. Poletti PA, Platon A, Rutschmann OT, Schmidlin FR, Iselin CE, Becker CD (2007) Low-dose versus standard-dose CT protocol in patients with clinically suspected renal colic. AJR Am J Roentgenol 188:927-933

12. Li J, Udayasankar UK, Toth TL, Seamans J, Small WC, Kalra MK (2007) Automatic patient centering for MDCT: effect on radiation dose. AJR Am J Roentgenol 188:547-552

13. Kalra MK, Maher MM, Toth TL et al (2004) Strategies for CT radiation dose optimization. Radiology 230:619-628

14. Alkadhi H, Leschka S (2011) Radiation dose of cardiac computed tomography - what has been achieved and what needs to be done. Eur Radiol 21:505-509

15. Schell B, Bauer RW, Lehnert T et al (2011) Low-dose computed tomography of the paranasal sinus and facial skull using a high-pitch dual-source system-first clinical results. Eur Radiol 21:107-112

16. Kazerooni E, Sundaram B, Hohenberg P, Hanlon D Availability, Awareness, and Use of Dose Reduction Technologies among Radiologists: The CT Awareness of Radiation Exposure Study (CARES). scientific presentation at RSNA meeting 2008

17. Shrimpton PC, Hiller MC, Lewis MA, Dunn M (2005) Doses from Computed Tomography (CT) examinations in the UK-2003. Available via http://www.hpa.org.uk/web/HPAwebFile/HPAweb_C/ 1194947420292. Accessed 14 April 2011

18. Treier R, Aroua A, Verdun FR, Samara E, Stuessi A, Trueb PR (2010) Patient doses in CT examinations in Switzerland: implementation of national diagnostic reference levels. Radiat Prot Dosimetry $142: 244-254$

19. Huda W, Ogden KM, Khorasani MR (2008) Converting doselength product to effective dose at CT. Radiology 248:995-1003

20. International Commission on Radiological Protection (2008) 2007 Recommendations of the International Commission on Radiological, Annals of the ICRP, ICRP Publication 103. Pergamon, Oxford 
21. Kalra MK, Maher MM, Blake MA et al (2004) Detection and characterization of lesions on low-radiation-dose abdominal CT images postprocessed with noise reduction filters. Radiology 232:791-797

22. Funama Y, Awai K, Miyazaki O et al (2006) Improvement of lowcontrast detectability in low-dose hepatic multidetector computed tomography using a novel adaptive filter: evaluation with a computer-simulated liver including tumors. Invest Radiol 41:1-7

23. Shin HO, Falck CV, Galanski M (2004) Low-contrast detectability in volume rendering: a phantom study on multidetector-row spiral CT data. Eur Radiol 14:341-349

24. von Falck C, Galanski M, Shin HO (2010) Informatics in radiology: sliding-thin-slab averaging for improved depiction of low-contrast lesions with radiation dose savings at thin-section CT. Radiographics 30:317-326

25. Brink JA, Amis ES Jr (2010) Image Wisely: a campaign to increase awareness about adult radiation protection. Radiology 257:601-602

26. Goske MJ, Applegate KE, Boylan J et al (2008) The Image Gently campaign: working together to change practice. AJR Am J Roentgenol 190:273-274

27. Raff GL, Chinnaiyan KM, Share DA et al (2009) Radiation dose from cardiac computed tomography before and after implementation of radiation dose-reduction techniques. JAMA 301:2340-2348

28. Wallace AB, Goergen SK, Schick D, Soblusky T, Jolley D (2010) Multidetector $\mathrm{CT}$ dose: clinical practice improvement strategies from a successful optimization program. J Am Coll Radiol 7:614-624

29. Hricak H, Brenner DJ, Adelstein SJ et al (2010) Managing radiation use in medical imaging: a multifaceted challenge. Radiology 258:889-905

30. Ertl-Wagner BB, Hoffmann RT, Bruning R et al (2004) Multi-detector row CT angiography of the brain at various kilovoltage settings. Radiology 231:528-535

31. Waaijer A, Prokop M, Velthuis BK, Bakker CJ, de Kort GA, van Leeuwen MS (2007) Circle of Willis at CT angiography: dose reduction and image quality-reducing tube voltage and increasing tube current settings. Radiology 242:832-839

32. Schueller-Weidekamm C, Schaefer-Prokop CM, Weber M, Herold CJ, Prokop M (2006) CT angiography of pulmonary arteries to detect pulmonary embolism: improvement of vascular enhancement with low kilovoltage settings. Radiology 241:899-907

33. Heyer CM, Mohr PS, Lemburg SP, Peters SA, Nicolas V (2007) Image quality and radiation exposure at pulmonary $\mathrm{CT}$ angiography with 100 - or $120-\mathrm{kVp}$ protocol: prospective randomized study. Radiology 245:577-583

34. Szucs-Farkas Z, Schaller C, Bensler S, Patak MA, Vock P, Schindera ST (2009) Detection of pulmonary emboli with CT angiography at reduced radiation exposure and contrast material volume: comparison of $80 \mathrm{kVp}$ and $120 \mathrm{kVp}$ protocols in a matched cohort. Invest Radiol 44:793-799

35. Wintersperger B, Jakobs T, Herzog P et al (2005) Aorto-iliac multidetector-row $\mathrm{CT}$ angiography with low $\mathrm{kV}$ settings: improved vessel enhancement and simultaneous reduction of radiation dose. Eur Radiol 15:334-341

36. Kalva SP, Sahani DV, Hahn PF, Saini S (2006) Using the K-edge to improve contrast conspicuity and to lower radiation dose with a 16-MDCT: a phantom and human study. J Comput Assist Tomogr 30:391-397

37. Schindera ST, Nauer C, Treier R et al (2010) Strategies for reducing the CT radiation dose. Radiologe 50:1120-1127

38. Loupatatzis C, Schindera S, Gralla J et al (2008) Whole-body computed tomography for multiple traumas using a triphasic injection protocol. Eur Radiol 18:1206-1214

39. Kekelidze M, Dwarkasing RS, Dijkshoorn ML, Sikorska K, Verhagen PC, Krestin GP (2010) Kidney and urinary tract imaging: triple-bolus multidetector CT urography as a one-stop shop-protocol design, opacification, and image quality analysis. Radiology 255:508-516

40. Schindera ST, Nelson RC, Toth TL et al (2008) Effect of patient size on radiation dose for abdominal MDCT with automatic tube current modulation: phantom study. AJR Am J Roentgenol 190: W100-105

41. Nauer CB, Kellner-Weldon F, Von Allmen G, Schaller D, Gralla J (2009) Effective doses from scan projection radiographs of the head: impact of different scanning practices and comparison with conventional radiography. AJNR Am J Neuroradiol 30:155-159

42. O'Daniel JC, Stevens DM, Cody DD (2005) Reducing radiation exposure from survey CT scans. AJR Am J Roentgenol 185:509-515

43. Smith AB, Dillon WP, Gould R, Wintermark M (2007) Radiation dose-reduction strategies for neuroradiology CT protocols. AJNR Am J Neuroradiol 28:1628-1632

44. Kalra MK, Maher MM, Toth TL, Kamath RS, Halpern EF, Saini S (2004) Radiation from "extra" images acquired with abdominal and/or pelvic CT: effect of automatic tube current modulation. Radiology 232:409-414

45. Brink M, de Lange F, Oostveen LJ et al (2008) Arm raising at exposure-controlled multidetector trauma CT of thoracoabdominal region: higher image quality, lower radiation dose. Radiology 249:661-670

46. Li J, Udayasankar UK, Toth TL, Small WC, Kalra MK (2008) Application of automatic vertical positioning software to reduce radiation exposure in multidetector row computed tomography of the chest. Invest Radiol 43:447-452

47. Matsubara K, Koshida K, Ichikawa K et al (2009) Misoperation of CT automatic tube current modulation systems with inappropriate patient centering: phantom studies. AJR Am J Roentgenol 192:862-865 